\title{
Epigallocatechin-3-gallate suppresses alveolar epithelial cell apoptosis in seawater aspiration-induced acute lung injury via inhibiting STAT1-caspase-3/p21 associated pathway
}

\author{
WEI LIU ${ }^{1 *}$, MINGQING DONG ${ }^{2 *}$, LIYAN BO $^{1}$, CONGCONG LI $^{1}$, \\ QINGQING LIU ${ }^{1}$, ZHICHAO LI $^{2}$ and FAGUANG JIN ${ }^{1}$ \\ ${ }^{1}$ Department of Pulmonary Diseases, Tangdu Hospital, Fourth Military Medical University, Xi'an, Shaanxi 710038; \\ ${ }^{2}$ Department of Pathology and Pathophysiology, Fourth Military Medical University, Xi'an, Shaanxi 710032, P.R. China
}

Received March 15, 2015; Accepted October 26, 2015

DOI: $10.3892 / \mathrm{mmr} .2015 .4617$

\begin{abstract}
The apoptosis of alveolar epithelial cells is important in seawater aspiration-induced acute lung injury (ALI). The present study aimed to investigate whether epigallocatechin-3-gallate (EGCG) is able to suppress apoptosis in alveolar epithelial cells in seawater aspiration-induced ALI in vivo and in vitro, and the possible mechanisms underlying it. The results indicated that seawater aspiration-induced ALI in rats is accompanied by increased apoptosis in lung tissue cells and the expression of apoptosis-associated proteins, caspase-3 and p21.EGCG pretreatment significantly ameliorated seawater aspiration-induced ALI. Furthermore, EGCG decreased seawater aspiration-induced apoptosis and the expression of caspase-3 and p21 in lung tissue cells. Seawater-challenged A549 cells experienced increased apoptosis and elevated levels of phosphorylated-signal transducer and activator of transcription 1 (P-STAT1). EGCG pretreatment of the cells resulted in significantly decreased seawater-induced apoptosis and lower levels of STAT1 and P-STAT1 in A549 cells. This suggests that EGCG suppresses alveolar epithelial cell apoptosis in seawater aspiration-induced ALI via inhibiting the STAT1-caspase-3/p21 associated pathway.
\end{abstract}

Correspondence to: Professor Faguang Jin, Department of Pulmonary Diseases, Tangdu Hospital, Fourth Military Medical University, 569 Xinsi Road, Xi'an, Shaanxi 710038, P.R. China

E-mail: jinfag@fmmu.edu.cn

Professor Zhichao Li, Department of Pathology and Pathophysiology, Fourth Military Medical University, 17 Changle Road, Xi'an, Shaanxi 710032, P.R. China

E-mail: lizhic@fmmu.edu.cn

*Contributed equally

Key words: seawater, acute lung injury, epigallocatechin-3-gallate, apoptosis, STAT1

\section{Introduction}

Drowning is one of the principal causes of accidental deaths, and thus presents a serious public health risk (1). Acute lung injury (ALI) caused by drowning is a critical complication that often results in mortality. Instances of seawater aspiration often lead to more severe ALI than those of freshwater aspiration, and thus present a higher mortality rate (2). Seawater aspiration-induced ALI is often characterized by acute inflammation of lung parenchyma and interstitial tissue with severe hypoxemia and pulmonary edema $(3,4)$. However, the apoptosis of alveolar epithelial cells can also result in high endothelial cell permeability and pulmonary edema in seawater aspiration-induced ALI $(5,6)$.

Epigallocatechin-3-gallate (EGCG), the main ingredient in green tea, has been reported to ameliorate seawater aspiration-induced ALI by regulating the inflammatory response in rats (7). EGCG may also trigger apoptosis in tumor tissues, due to the inactivation of $\mathrm{NF}-\kappa \mathrm{B}$ and the decreased expression of cyclin D1 $(8,9)$. Conversely, EGCG may protect against ischemia/reperfusion-induced apoptosis and cisplatin-induced apoptosis in heart and kidney $(10,11)$. Therefore, the current study aimed to determine the effect of EGCG on alveolar epithelial cells exposed to seawater aspiration-induced ALI.

EGCG has been reported to reduce signal transducer and activator of transcription 1 (STAT1) phosphorylation in various human cells (12). EGCG may reduce inflammation and pulmonary edema in seawater aspiration-induced ALI via inhibiting the JAK/STAT1 pathway (7). Furthermore, inhibiting STAT1 may reduce cerebral and osteocyte cell apoptosis $(13,14)$. Therefore, EGCG may also be capable of suppressing alveolar epithelial cell apoptosis in seawater aspiration-induced ALI via inhibiting the STAT1-caspase-3/p21 pathway.

\section{Materials and methods}

Ethical approval. All experimental procedures were authorized by the Animal Care and Use Committees of the Fourth Military Medical University (Xi'an, China) and followed the protocols outlined in the Guide for Care and Use of Laboratory 
Animals published by the National Institutes of Health (publication no. 85-23, revised 1985).

Chemicals and reagents. EGCG (purity >99\%) was purchased from Sigma-Aldrich (St. Louis, MO, USA) and prepared as a stock solution in normal saline. Seawater [osmolality $1,300 \mathrm{mmol} / \mathrm{l}(\mathrm{pH} 8.2)$, specific weight 1.05 ; $\mathrm{NaCl}, 26.518 \mathrm{~g} / \mathrm{l} ; \mathrm{MgSO}_{4}, 3.305 \mathrm{~g} / \mathrm{l} ; \mathrm{MgCl}_{2}, 2.447 \mathrm{~g} / \mathrm{l} ; \mathrm{CaCl}_{2}$, $1.141 \mathrm{~g} / \mathrm{l} ; \mathrm{KCl}, 0.725 \mathrm{~g} / \mathrm{l} ; \mathrm{NaHCO}_{3}, 0.202 \mathrm{~g} / \mathrm{l}$; and $\mathrm{NaBr}$, $0.083 \mathrm{~g} / \mathrm{l}$; all reagents from Sigma-Aldrich) was prepared in order to mimic the chemical composition of the East China Sea, provided by the Chinese Ocean Bureau (Beijing, China). STAT1 (cat. no. 9172) and phospho-STAT1-Tyr-701 (cat. no. 9167) rabbit monoclonal antibodies were purchased from Cell Signaling Technology, Inc. (Danvers, MA, USA). p21 (cat. no. sc-271532) and $\beta$-actin (cat. no. sc-8432) mouse monoclonal antibodies were purchased from Santa Cruz Biotechnology, Inc. (Dallas, TX, USA). Cleaved caspase-3 (cat. no. a0214) rabbit monoclonal antibody was purchased from ABclonal Biotech Co., Ltd. (Cambridge, MA, USA). An annexin V/fluorescein isothiocyanate (FITC) kit was purchased from BD Biosciences (San Jose, CA, USA). A TUNEL In Situ Cell Death Detection Kit, Fluorescein was purchased from Roche Diagnostics (Laval, Canada). Evans Blue was obtained from Sigma-Aldrich. The Total Protein Extraction kit and the bicinchoninic acid protein assay kit were supplied by Thermo Fisher Scientific, Inc. (Waltham, MA, USA).

Animals and groups. The seawater aspiration-induced ALI rat model used in the current study followed the procedure outlined in Liu et al (7). Prior to exposure of the trachea, the rats were anesthetized with $3 \%$ pentobarbital sodium $[1.5 \mathrm{ml} / \mathrm{kg}$, administered by intraperitoneal (i.p.) injection]. A total of 24 adult male Sprague-Dawley rats (180-220 g) were obtained from the Animal Center of the Fourth Military Medical University. They were randomly divided into four groups (all $n=6$ ) as follows: (i) The control group, no treatment was applied, only the trachea was exposed; (ii) the seawater-only group, seawater ( $4 \mathrm{ml} / \mathrm{kg}$ ) was instilled into rat lungs following trachea exposure within $2 \mathrm{~min}$, maintaining a constant speed using a $1 \mathrm{ml}$ syringe; (iii) the seawater + EGCG group, EGCG $(10 \mathrm{mg} / \mathrm{kg}$; i.p. injection) $30 \mathrm{~min}$ prior to seawater exposure; and (iv) the EGCG-only group, EGCG (10 mg/kg; i.p. injection) was injected prior to trachea exposure. The rats were sacrificed by aortic transection $6 \mathrm{~h}$ subsequent to treatment. The dosage of EGCG used was determined on the basis of a previous study (15).

Lung edema and microvascular permeability. The wet-to-dry weight ratio of the lung was as an indication of lung edema. The upper lobes of the right lungs were obtained $6 \mathrm{~h}$ following application of experimental treatment and weighed immediately subsequent to removal, then subjected to desiccation at $55^{\circ} \mathrm{C}$ for $72 \mathrm{~h}$ and weighed again. The ratio of wet-to-dry was calculated by dividing the wet weight by the dry weight (16).

Microvascular permeability was examined by the extravasation of Evans Blue into the tissue. Evans Blue $(20 \mathrm{mg} / \mathrm{kg})$ was administered through a tail vein $0.5 \mathrm{~h}$ prior to euthanasia. The pulmonary circulation was flushed with $10 \mathrm{ml}$ phosphate-buffered saline (PBS; Sigma-Aldrich). The lungs were subsequently excised and frozen in liquid nitrogen. The frozen tissue was homogenized and incubated with formamide at $60^{\circ} \mathrm{C}$ for $16 \mathrm{~h}$, prior to centrifugation at 7,000 x g for $20 \mathrm{~min}$. The absorbance (A620 and A740) of the supernatant was measured using a spectrophotometer [model no. 722; Suoyu (Shanghai) Electronic Co., Ltd., Shanghai, China], and the Evans Blue content ( $\mu \mathrm{g} / \mathrm{g}$ lung) was calculated against the generated Evans Blue standard absorbance curves (5).

Histopathology. At the end of the experiments, lung tissues of the same lobe from each rat were fixed with $10 \%$ formalin, embedded in paraffin and stained with hematoxylin-eosin. The different group tissue sections were blindly assessed by two independent pathologists. They were scored based on edema, neutrophil infiltration, hemorrhage, bronchiole epithelial desquamation and hyaline membrane formation. A scale of 0-4 indicated the severity of the lung tissue injury as follows: 0, no injuries (normal in appearance); 1 , limited injuries; 2 , intermediate injuries; 3 , widespread injuries; and 4 , prominent injuries (17).

Quantification of apoptosis in lung tissue sections. The apoptotic cells were identified using the TUNEL kit, which was conducted in accordance with the manufacturer's protocol. Apoptotic cells were counted under a fluorescence microscope (Eclipse Ti-SR; Nikon Corp., Tokyo, Japan). A total of 6 tissue sections from each group were randomly selected, and 10 fields were evaluated per section at x200 magnification. Image Pro Plus, version 6.0 software (Media Cybernetics, Inc., Rockville, MD, USA) was used to obtain the average number of apoptotic cells per visual field.

Cell culture and treatment. Human lung alveolar epithelial cells (A549) were obtained from the American Type Culture Collection (Rockville, MD, USA). Cells were cultured in HyClone ${ }^{\mathrm{TM}}$ RPMI 1640 medium (GE Healthcare Life Sciences, Logan, Utah, USA) supplemented with $10 \%$ fetal bovine serum (Gibco BRL, Gaithersburg, MD, USA) at $37^{\circ} \mathrm{C}$ in a humidified atmosphere with $5 \% \mathrm{CO}_{2}$. In order to induce apoptosis, the following concentrations of seawater were administered for $6 \mathrm{~h}$ : 10, 20, 30 and $40 \%$, equivalent to $0.1,0.2,0.3$ and $0.4 \mathrm{ml}$ per $1 \mathrm{ml}$ total volume. The effect of EGCG on A549 cells treated with seawater was also examined. Two treatment groups were established as follows: i) The seawater-only group, cells were exposed to $30 \%$ seawater for $6 \mathrm{~h}$; and ii) the seawater + EGCG group, cells were initially treated with $10 \mu \mathrm{M}$ EGCG for $30 \mathrm{~min}$ and then exposed to the 6 -h $30 \%$ seawater treatment.

Flow cytometry. The percentage of apoptotic A549 cells in each group was determined using annexin V-FITC and propidium iodide (PI) staining and analyzed by flow cytometric analysis. Following digestion, the cells were centrifuged at $1,000 \mathrm{x}$ for $5 \mathrm{~min}$. They were then washed three times with PBS and resuspended in $400 \mu l$ annexin binding buffer. Subsequently, PI and FITC-conjugated annexin V were added, and the cell suspension was incubated for $15 \mathrm{~min}$ in the dark prior to analysis using a FACSCalibur flow cytometer (BD FACSAria ${ }^{\mathrm{TM}}$ III; BD Biosciences) (6). 
Table I. Lung injury scores in each group.

\begin{tabular}{|c|c|c|c|c|}
\hline Group & Neutrophil infiltration & Edema & Hemorrhage & Epithelial desquamation \\
\hline Control & $0.2 \pm 0.16$ & $0.3 \pm 0.14$ & $0.2 \pm 0.18$ & $0.1 \pm 0.09$ \\
\hline Seawater & $3.5 \pm 0.28^{\mathrm{a}}$ & $3.4 \pm 0.31^{\mathrm{a}}$ & $3.1 \pm 0.25^{\mathrm{a}}$ & $3.4 \pm 0.12^{\mathrm{a}}$ \\
\hline Seawater + EGCG & $1.9 \pm 0.24^{\mathrm{b}}$ & $2.0 \pm 0.23^{b}$ & $1.8 \pm 0.31^{\mathrm{b}}$ & $1.4 \pm 0.25^{\mathrm{b}}$ \\
\hline EGCG & $0.3 \pm 0.23$ & $0.2 \pm 0.13$ & $0.2 \pm 0.12$ & $0.2 \pm 0.17$ \\
\hline
\end{tabular}

Data are presented as the mean \pm standard error; ${ }^{a} \mathrm{P}<0.05$ vs. the control group and ${ }^{b} \mathrm{P}<0.05$ vs. seawater group; $\mathrm{n}=8$. EGCG, epigallocatechin-3-gallate.

A

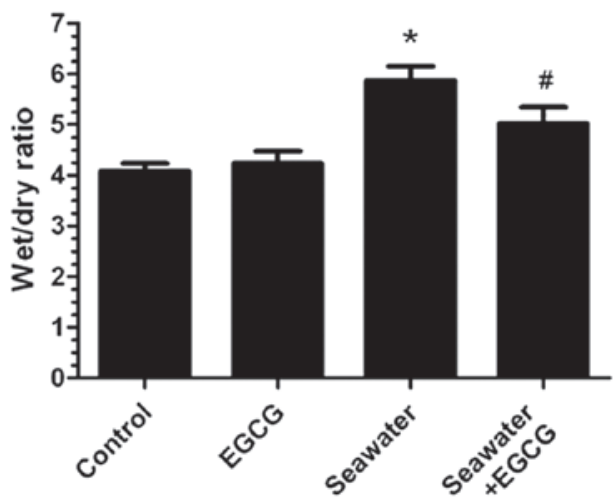

B

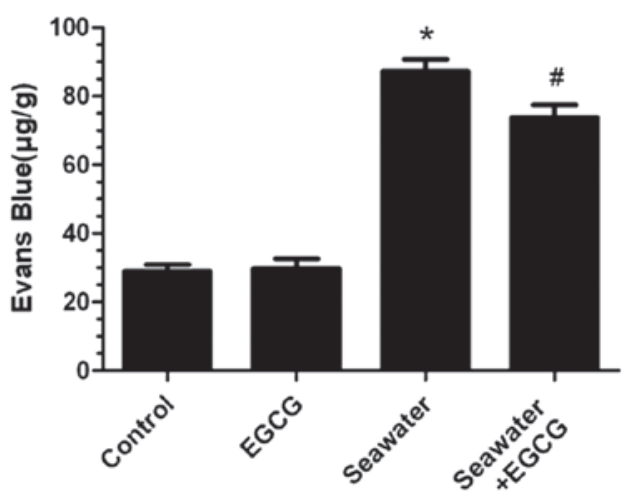

Figure 1. Effects of EGCG on the lung edema and vascular leakage in seawater aspiration-induced acute lung injury. (A) Lung wet-to-dry weight ratio for the varying treatments $(n=6)$. (B) Leak index of Evans Blue $(n=6)$. Data are presented as the mean \pm standard error. $(n=6)$. $P<0.05$ vs. the control group, ${ }^{\#} \mathrm{P}<0.05$ vs. the seawater group. EGCG, epigallocatechin-3-gallate.

Western blot analysis. Part of the right lung tissue from the experimental rats was sampled along with A549 cells for each treatment group. Total protein was extracted from whole-cell and lung tissues and prepared according to the manufacturer's protocol with a Total Protein Extraction kit. Protein concentrations were identified by bicinchoninic acid protein assay kit. The samples were then separated in parallel with $8 \%$ gradient (spacer gel, $80 \mathrm{~V}$; separation gel, $120 \mathrm{~V}$ ), loaded onto a SDS-PAGE gel (Beyotime Institute of Biotechnology, Shanghai, China) and transferred to an Invitrogen ${ }^{\mathrm{TM}}$ nitrocellulose membrane (Thermo Fisher Scientific). Membranes were blocked for $2 \mathrm{~h}$, prior to overnight incubation at $4^{\circ} \mathrm{C}$ with the relevant primary antibodies against STAT1 (1:500), P-STAT1 (1:500), caspase-3 (1:1,000), p21 (1:1,000) and $\beta$-actin $(1: 1,000)$. The membranes were then washed five times and incubated for $2 \mathrm{~h}$ with the secondary antibody (anti-mouse or anti-rabbit, 1:5,000; cat nos. 5571 and 7076; Cell Signaling Technology, Inc.). Detection was performed using a chemiluminescence system (ImageQuant LAS 4000 Mini; GE Healthcare Life Sciences, Chalfont, UK).

Statistical analysis. Data are expressed as the mean \pm standard error and statistical analysis was performed with one-way analysis of variance, followed by a Dunnett's test for multiple comparisons. As the histological injury score data is not continuous, the non-parametric, Kruskal-Wallis one-way analysis of variance was used. $\mathrm{P}<0.05$ was considered to indicate a statistically significant difference.

\section{Results}

Effects of EGCG on lung edema and microvascular permeability in seawater aspiration-induced ALI. The extent of lung edema and microvascular permeability was evaluated using the lung wet-to-dry weight ratios and the leak index. As demonstrated by Fig. 1, the wet-to-dry lung ratio was significantly greater following seawater aspiration compared with the control group (5.87 \pm 0.28 vs. $4.08 \pm 0.15 ; \mathrm{P}<0.05 ; \mathrm{n}=6)$, whilst the EGCG pretreatment reduced the lung wet-to-dry weight ratio compared with the seawater-only group $(5.02 \pm 0.33$ vs. $5.87 \pm 0.28 ; \mathrm{P}<0.05 ; \mathrm{n}=6$ ). In the seawater-only group, the leak of Evans Blue was significantly greater compared with the control group (87.2 \pm 3.61 vs. $28.98 \pm 1.90 ; \mathrm{P}<0.05 ; \mathrm{n}=6)$. The EGCG pretreatment had a lower leak index compared with the control group (73.8 \pm 3.63 vs. 87.2 $\pm 3.61 ; \mathrm{P}<0.05 ; \mathrm{n}=6)$.

Effects of EGCG on histopathological changes and the lung injury scores in seawater aspiration-induced ALI. As demonstrated by Fig. 2, seawater aspiration induced lung edema, causing inflammatory cells to infiltrate the lung tissues and alveoli, resulting in hemorrhage, bronchiole epithelial desquamation and alveolar collapse (Fig. 2B). EGCG pretreatment ameliorated these histopathological changes (Fig. 2C). The lung injury scores of each group are presented in Table I. These results indicated that EGCG can reduce the lung injury scores in seawater aspiration-induced ALI. 
A

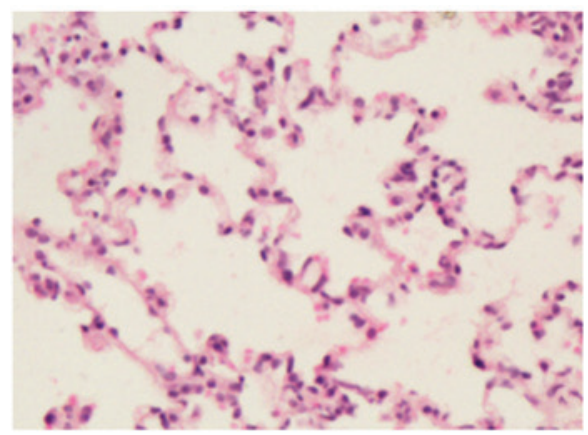

B

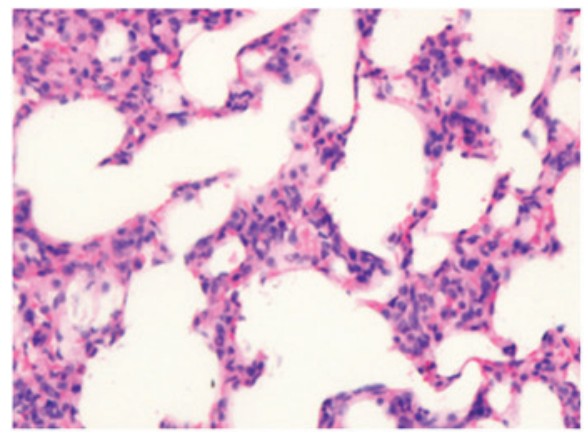

C

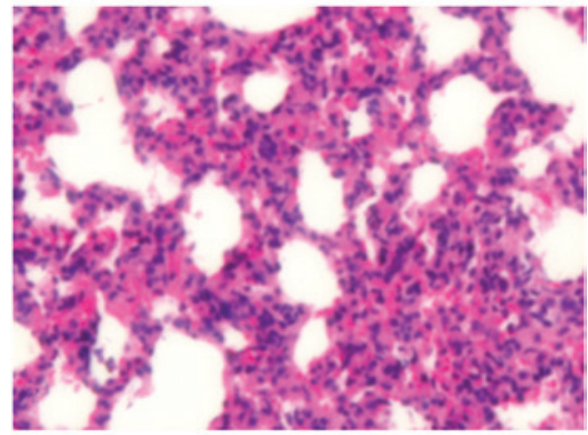

D

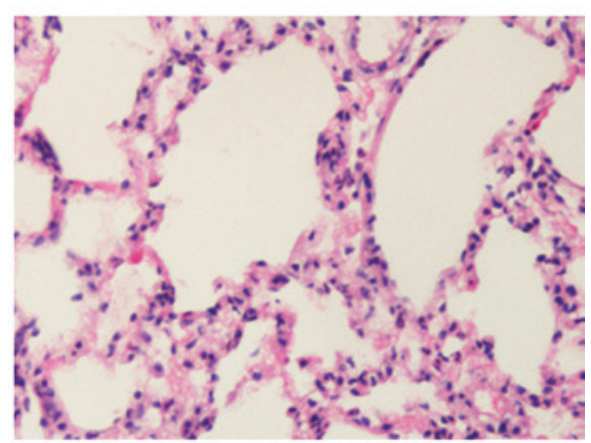

Figure 2. Effects of EGCG on the histopathological changes in seawater aspiration-induced acute lung injury. The (A) control, (B) seawater, (C) seawater + EGCG and (D) EGCG groups. Hematoxylin and eosin staining, magnification x200. EGCG, epigallocatechin-3-gallate.

Effects of EGCG on apoptosis in seawater aspiration-induced $A L I$. Fluorescence TUNEL staining on lung sections from each group determined the effect of EGCG on the apoptosis of seawater aspiration-induced ALI (Fig. 3A). The number of fluorescent TUNEL-positive cells significantly increased in the seawater-only group compared with the control group $(\mathrm{P}<0.05$; Fig. 3B). EGCG pretreatment reduced the fluorescence of the
A

\section{Negative control}

Control

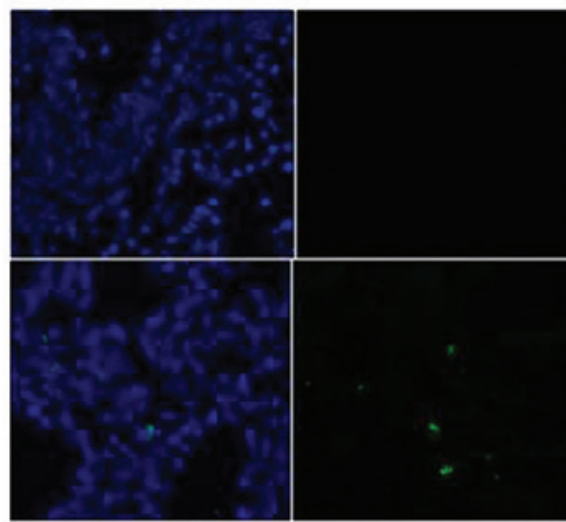

EGCG

Seawater

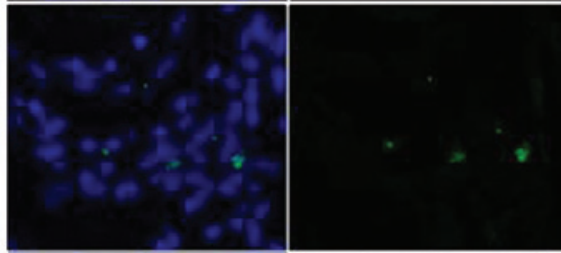

Seawater +EGCG

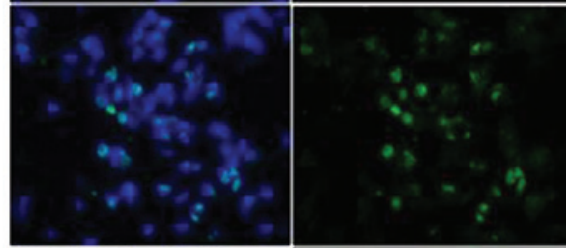

B

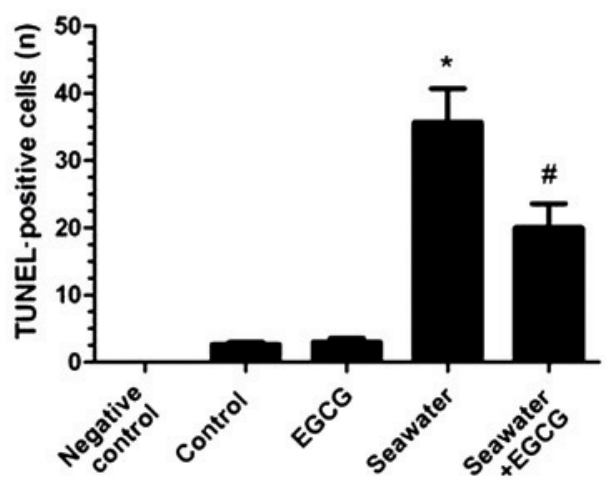

Figure 3. Effects of EGCG on the apoptosis in lungs in seawater aspiration-induced acute lung injury. (A) Fluorescence TUNEL staining of lung section from each group. (B) Quantified data of TUNEL-positive cells. Data are presented as the mean \pm standard error; ${ }^{*} \mathrm{P}<0.05$ vs. the control group and ${ }^{\#} \mathrm{P}<0.05$ vs. the seawater group; $\mathrm{n}=6$. Magnification, $\mathrm{x} 200$. EGCG, epigallocatechin-3-gallate.

TUNEL-positive cells compared with the seawater group $(\mathrm{P}<0.05$; Fig. 3B). No significant differences were identified between the control and EGCG groups.

Effects of EGCG on the expression of apoptosis-associated proteins in seawater aspiration-induced ALI. Levels of the apoptosis-associated proteins caspase- 3 and p21 in the 
A

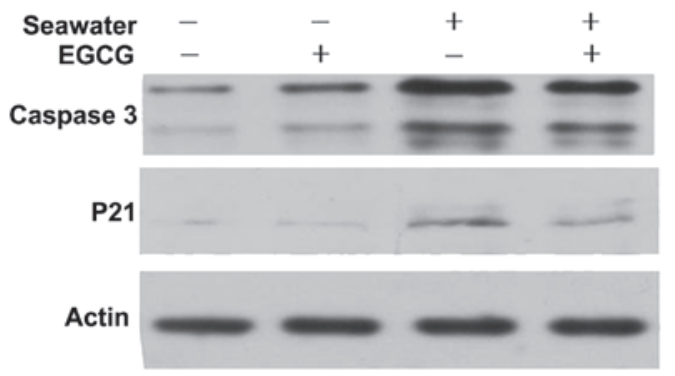

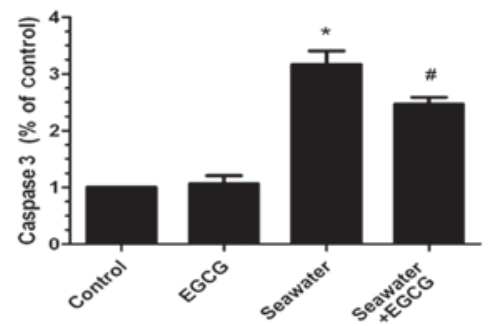

C

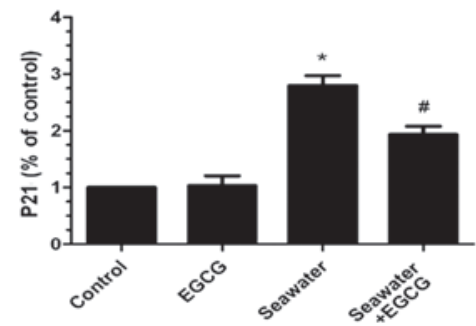

Figure 4. Effects of EGCG on the expression of caspase-3 and p21 in lungs in seawater aspiration-induced acute lung injury. (A) Western blot for the caspase-3 and $\mathrm{p} 21$ protein expression in the different groups. Quantified western blot data for (B) caspase-3 and (C) p21. Data represent three independent experiments and are expressed as the mean \pm standard error. ${ }^{*} \mathrm{P}<0.05$ vs. the control group and ${ }^{\#} \mathrm{P}<0.05$ vs. the seawater group. EGCG, epigallocatechin-3-gallate.

A

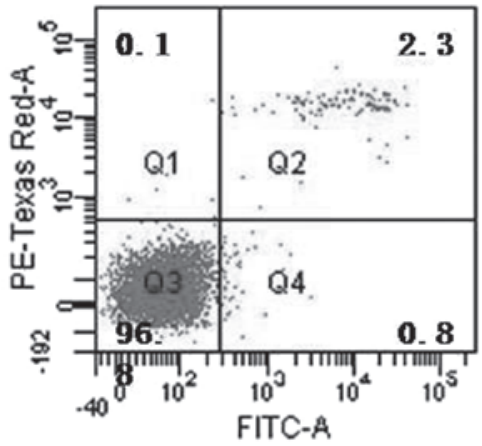

D

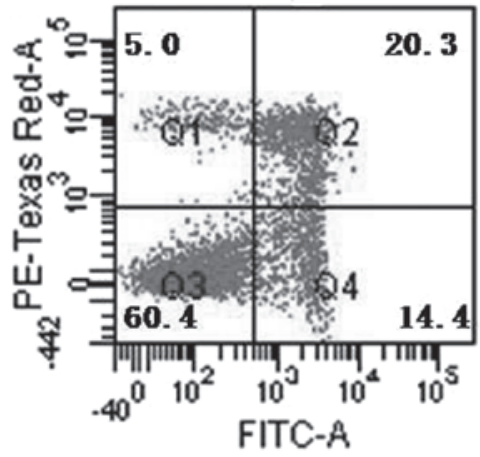

B

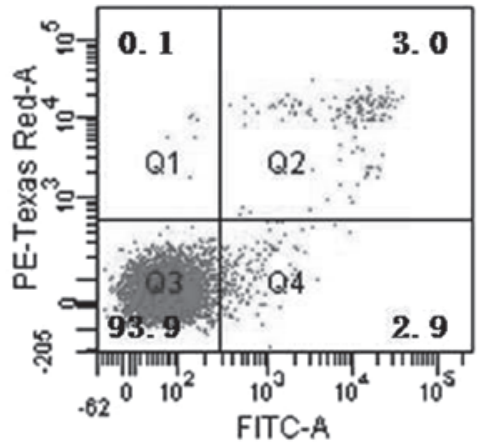

$\mathbf{E}$

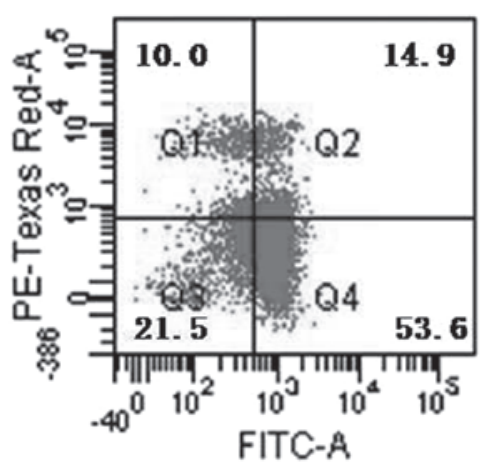

C

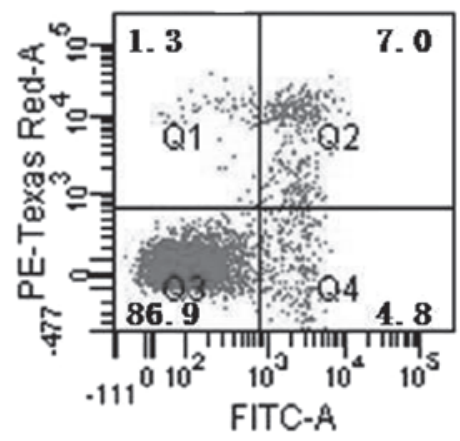

$\mathbf{F}$

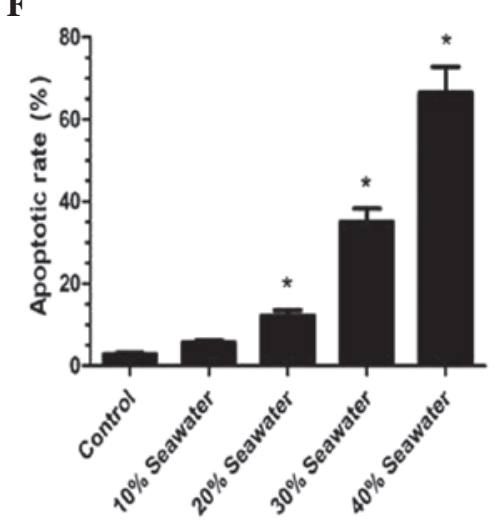

Figure 5. Effects of different concentrations of seawater on apoptosis in A549 cells. The (A) control, (B) 10\% seawater, (C) $20 \%$ seawater, (D) 30\% seawater and (E) $40 \%$ seawater groups. (F) Quantified rates of apoptosis in each group. Data are presented as the mean \pm standard error. ${ }^{*} \mathrm{P}<0.05$ vs. the control group; $\mathrm{n}=4$. FITC, fluorescein isothiocyanate; PE-Texas Red-A, R-phycoerythrin-Texas Red.

lungs of the rats that had been exposed to seawater were assessed by western blot analysis (Fig. 4A). The results demonstrated that caspase- 3 and p21 expression levels were increased in the seawater-only group $(\mathrm{P}<0.05$ vs. the control group; Fig. 4A and B), whilst expression was significantly decreased in the seawater + EGCG group $(\mathrm{P}<0.05$ vs. the seawater group; Fig. $4 \mathrm{~B}$ and $\mathrm{C}$ ). No significant differences were identified between the seawater + EGCG and control groups.

Effects of EGCG on the seawater-induced apoptosis of the A549 cells. The effects of EGCG on the apoptosis of alveolar epithelial cell line A549 when treated with seawater were explored. As shown in Fig. 5, the percentage of apoptotic A549 cells increased in a concentration-dependent manner.
The apoptotic rate reached $68.5 \%$ in the $40 \%$ seawater group $(\mathrm{P}<0.05$ vs. the control group; Fig. 5F). However, no significant difference between the control group and $10 \%$ seawater group was identified.

The effect of EGCG on A549 cells treated with $30 \%$ saltwater was further investigated. The EGCG pretreatment significantly decreased the percentage of A549 cells undergoing apoptosis $(\mathrm{P}<0.05$ vs. the control group; Fig. 6). No significant differences were identified between the percentage of cells undergoing apoptosis in the control group and the EGCG-only group (P>0.05; Fig. 6).

Effects of seawater and EGCG on the expression of STAT1 and P-STAT1 in A549 cells. The effects of seawater and EGCG on the expression of STAT1, a key upstream 
A

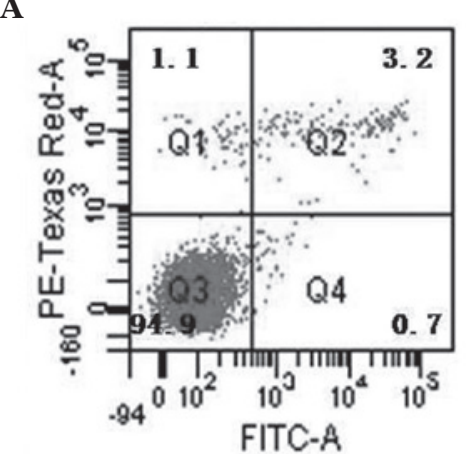

C

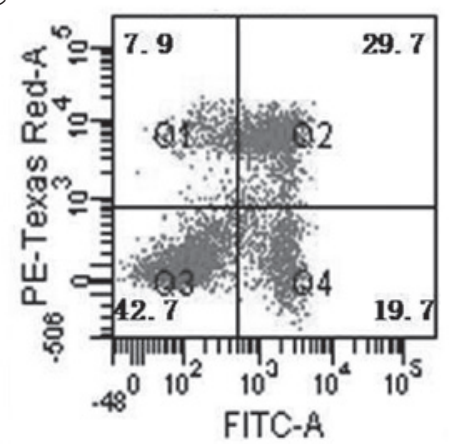

B

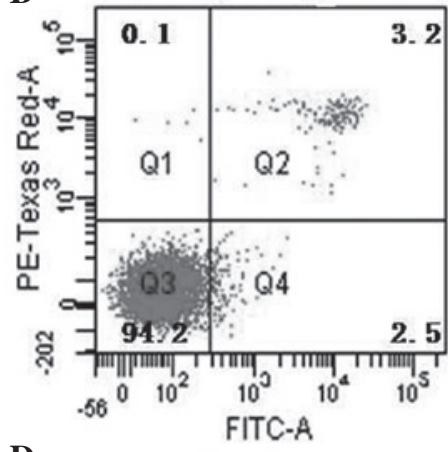

D

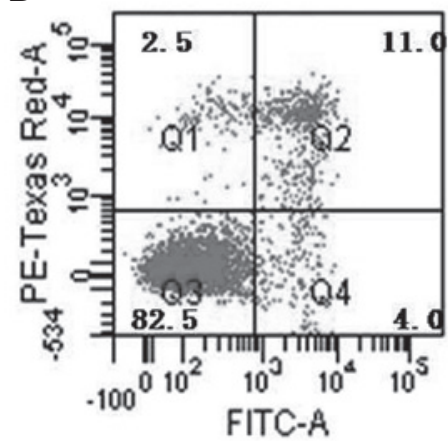

$\mathbf{E}$

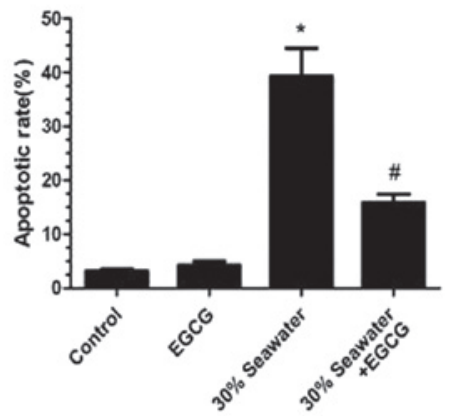

Figure 6. Effects of EGCG on apoptosis in A549 cells in the varying treatment groups. (A) Control group, (B) EGCG-only group, (C) $30 \%$ seawater-only group. and (D) EGCG $+30 \%$ seawater group. (E) Quantified data of apoptotic rate of each treatment group. Data are presented as the mean \pm standard error ${ }^{*} \mathrm{P}<0.05$ vs. control group, ${ }^{\text {"P}} \mathrm{P}<0.05$ vs. seawater group, $\mathrm{n}=4$. EGCG, epigallocatechin-3-gallate; PE-Texas Red-A, R-phycoerythrin; FITC, fluorescein isothiocyanate.

A

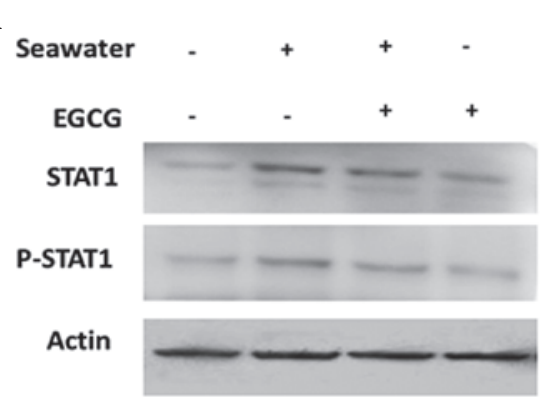

$\mathbf{C}$

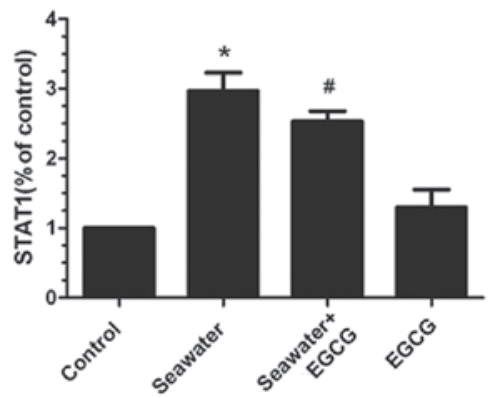

B

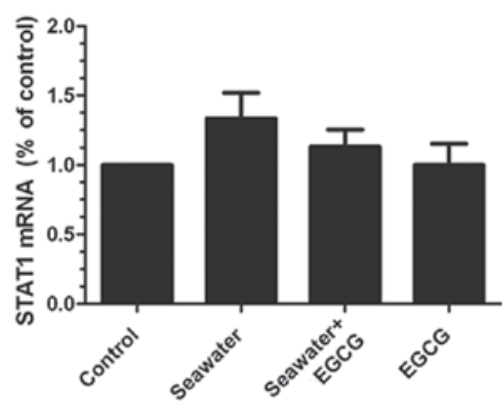

D

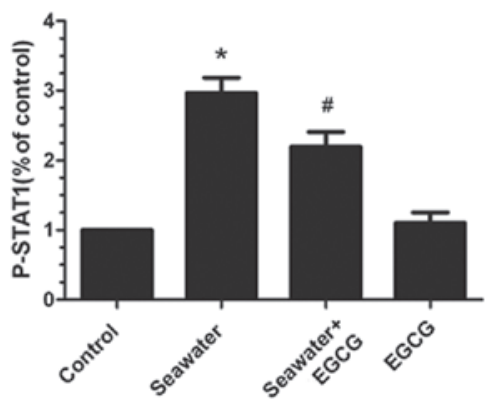

Figure 7. Effects of EGCG on the expression of STAT1 and P-STAT1 in A549 cells in the presence and absence of 30\% seawater. (A) Western blot for the STAT1 and P-STAT1 protein expression in the different treatment groups. (B) mRNA expression of STAT1 in A549 cells. (C) STAT1 and (D) P-STAT1 protein expression levels. Data represent three independent experiments and are expressed as the mean \pm standard error; ${ }^{*} \mathrm{P}<0.05$ vs. the control group and ${ }^{\#} \mathrm{P}<0.05$ vs. the seawater group. EGCG, epigallocatechin-3-gallate; STAT1, signal transducer and activator of transcription 1; P-STAT1, phosphorylated-STAT1.

modulator of caspase-3 and p21, was examined in A549 cells. No significant differences were identified in the STAT1 mRNA expression levels between any of the treatment groups (Fig. 7A and B). However, the protein expression levels of STAT1 and P-STAT1 were significantly elevated in the seawater-only group compared with the control group $(\mathrm{P}<0.05$; Fig. 7C and D). The group pretreated with EGCG presented significantly reduced protein expression levels of STAT1 and P-STAT1 compared with the seawater-only group $(\mathrm{P}<0.05$; Fig. $7 \mathrm{C}$ and $\mathrm{D})$. 


\section{Discussion}

In the present study, it was demonstrated that EGCG pretreatment alleviated seawater aspiration-induced ALI. EGCG pretreatment decreased the apoptosis of alveolar epithelial cells in seawater aspiration-induced ALI. To further investigate the role of EGCG in the apoptosis of alveolar epithelium in seawater aspiration-induced ALI, the expression levels of caspase-3 and p21 were determined, as they are apoptosis-associated proteins. Notably, protein levels were significantly higher in seawater aspiration-induced ALI compared with the control group. By contrast, the EGCG pretreatment group exhibited significantly lower levels of the apoptotic proteins, suggesting that EGCG may limit the damage that cells undergo during seawater aspiration-induced ALI. The expression of the protein STAT1 and phosphorylated STAT1, a key upstream protein of caspase-3 and p21, was identified to increase in the seawater-only group. However, protein expression levels of STAT1 and P-STAT1 decreased in the EGCG pretreatment group compared with the seawater group.

The alveolar epithelium and the microvascular endothelium constitute the alveolar-capillary barrier (18). The epithelial barrier is less permeable than the endothelial barrier under physiological conditions (19). The loss of integrity of alveolar epithelium is an important pathophysiological change during ALI, which leads to pulmonary edema and a decrease in surfactant-associated proteins $(18,20)$. The apoptosis of alveolar epithelial cells is one of the main factors damaging the alveolar-capillary barrier and thus, represents an important prognostic marker of ALI (21). Seawater aspiration-induced ALI is one of the most serious complications due to seawater drowning (22). Previous studies have indicated that the apoptosis of alveolar epithelium aggravated seawater aspiration-induced ALI, which may also be mediated via the Fas/FasL (6) and protein kinase (ERK) pathways (5).

In previous studies, EGCG has been demonstrated to exhibit anti-inflammatory (23), anti-oxidative (24) and anticancer (25) properties. In addition, EGCG has been indicated to have a positive effect on cell cycle arrest and apoptosis in prostate and breast cancer cells (26). Furthermore, it has been demonstrated to enhance cisplatin chemosensitivity in cervical cancer cells via the induction of apoptosis (27). Other studies have indicated that EGCG inhibits $\mathrm{Cd}^{2+}$-induced apoptosis through scavenging reactive oxygen species activity (28), and prevents cardiac ischemia/reperfusion-induced apoptosis (10). The findings of another previous study suggest that EGCG ameliorates inflammation in seawater aspiration-induced ALI by inhibiting the JAK/STAT1 pathways (7). However, it remains largely unknown whether EGCG protection during seawater aspiration-induced ALI is also mediated by the suppression of apoptosis in alveolar epithelial cells.

In the current study, it was determined that EGCG pretreatment attenuated the degree of ALI and inhibited the apoptosis of lung tissue and alveolar epithelial cells in vivo and in vitro. Simultaneously, EGCG pretreatment reduced the expression of the proteins STAT1, P-STAT1 and the downstream proteins caspase-3 and $\mathrm{p} 21$. Overexpression of STAT1 may induce osteocyte apoptosis in steroid-induced avascular necrosis of the femoral head and promote the apoptosis of retinal pericytes under high glucose conditions (29). The apoptosis-associated proteins caspase- 3 and p21, as the downstream proteins of STAT1, promoted the apoptosis of alveolar epithelial cells in seawater aspiration-induced ALI. Therefore, EGCG may inhibit the apoptosis of alveolar epithelial cells in seawater aspiration-induced ALI via decreasing the expression of STAT1, P-STAT1 and its downstream proteins caspase-3 and $\mathrm{p} 21$.

In summary, the present study suggests that EGCG attenuates seawater aspiration-induced ALI by suppressing the apoptosis of alveolar epithelial cells, at least partly, via the inhibition of the STAT1-caspase-3/p21 associated pathway.

\section{Acknowledgements}

The present study was supported by the National Natural Science Foundation of China (nos. 81270124 and 81372129).

\section{References}

1. Salomez F and Vincent JL: Drowning: A review of epidemiology, pathophysiology, treatment and prevention. Resuscitation 63: 261-268, 2004.

2. Rui M, Duan YY, Wang HL, Zhang XH and Wang Y: Differences between seawater- and freshwater-induced lung injuries. Zhongguo Wei Zhong Bing Ji Jiu Yi Xue 21: 416-420, 2009 (In Chinese).

3. Li J, Xu M, Fan Q, Xie X, Zhang Y, Mu D, Zhao P, Zhang B, Cao F, Wang Y, et al: Tanshinone IIA ameliorates seawater exposure-induced lung injury by inhibiting aquaporins (AQP) 1 and AQP5 expression in lung. Respir Physiol Neurobiol 176: 39-49, 2011

4. Ma L, Li Y, Zhao Y, Wang Q, Nan Y, Mu D, Li W, Sun R, Jin F and Liu X: 3,5,4'-tri-O-acetylresveratrol ameliorates seawater exposure-induced lung injury by upregulating connexin 43 expression in lung. Mediators Inflamm 2013: 182132, 2013.

5. Li JH, Xu M, Xie XY, Fan QX, Mu DG, Zhang Y, Cao FL, Wang YX, Zhao PT, Zhang B, et al: Tanshinone IIA suppresses lung injury and apoptosis, and modulates protein kinase B and extracellular signal-regulated protein kinase pathways in rats challenged with seawater exposure. Clin Exp Pharmacol Physiol 38: 269-277, 2011.

6. Han F, Luo Y, Li Y, Liu Z, Xu D, Jin F and Li Z: Seawater induces apoptosis in alveolar epithelial cells via the Fas/FasL-mediated pathway. Respir Physiol Neurobiol 182: 71-80, 2012.

7. Liu W, Dong M, Bo L, Li C, Liu Q, Li Y, Ma L, Xie Y, Fu E, $\mathrm{Mu} \mathrm{D}$, et al: Epigallocatechin-3-gallate ameliorates seawater aspiration-induced acute lung injury via regulating inflammatory cytokines and inhibiting JAK/STAT1 pathway in rats. Mediators Inflamm 2014: 612593, 2014.

8. Aggarwal BB and Shishodia S: Molecular targets of dietary agents for prevention and therapy of cancer. Biochem Pharmacol 71: 1397-1421, 2006.

9. ShankarS,SuthakarGandSrivastavaRK:Epigallocatechin-3-gallate inhibits cell cycle and induces apoptosis in pancreatic cancer. Front Biosci 12: 5039-5051, 2007.

10. Townsend PA, Scarabelli TM, Pasini E, Gitti G, Menegazzi M, Suzuki H, Knight RA, Latchman DS and Stephanou A: Epigallocatechin-3-gallate inhibits STAT-1 activation and protects cardiac myocytes from ischemia/reperfusion-induced apoptosis. FASEB J 18: 1621-1623, 2004.

11. Zou P, Song J, Jiang B, Pei F, Chen B, Yang X, Liu G and $\mathrm{Hu} \mathrm{Z}$ : Epigallocatechin-3-gallate protects against cisplatin nephrotoxicity by inhibiting the apoptosis in mouse. Int J Clin Exp Pathol 7: 4607-4616, 2014.

12. Menegazzi M, Tedeschi E, Dussin D, De Prati AC, Cavalieri E, Mariotto S and Suzuki H: Anti-interferon gamma action of epigallocatechin-3-gallate mediated by specific inhibition of STAT1 activation. FASEB J 15: 1309-1311, 2001.

13. Song CG, Yang X, Min LQ, Liu CX and Zhao CS: The effect of procyanidin on expression of STAT1 in type 2 diabetes mellitus SD rats with focal cerebral ischemia. Neuro Endocrinol Lett 35: 68-72, 2014. 
14. Xu X, Wen H, Hu Y, Yu H, Zhang Y, Chen C and Pan X: STAT1-caspase 3 pathway in the apoptotic process associated with steroid-induced necrosis of the femoral head. J Mol Histol 45: 473-485, 2014.

15. Bae HB, Li M, Kim JP, Kim SJ, Jeong CW, Lee HG, Kim WM, Kim HS and Kwak SH: The effect of epigallocatechin gallate on lipopolysaccharide-induced acute lung injury in a murine model. Inflammation 33: 82-91, 2010.

16. Shi Y, Zhang B, Chen XJ, Xu DQ, Wang YX, Dong HY, Ma SR, Sun RH, Hui YP and Li ZC: Osthole protects lipopolysaccharide-induced acute lung injury in mice by preventing down-regulation of angiotensin-converting enzyme 2. Eur J Pharm Sci 48: 819-824, 2013

17. Zhou ZH, Sun B, Lin K and Zhu LW: Prevention of rabbit acute lung injury by surfactant, inhaled nitric oxide, and pressure support ventilation. Am J Respir Crit Care Med 161: 581-588, 2000.

18. Ware LB and Matthay MA: The acute respiratory distress syndrome. N Engl J Med 342: 1334-1349, 2000.

19. Wiener-Kronish JP, Albertine KH and Matthay MA: Differential responses of the endothelial and epithelial barriers of the lung in sheep to Escherichia coli endotoxin. J Clin Invest 88: 864-875, 1991.

20. Greene KE, Wright JR, Steinberg KP, Ruzinski JT, Caldwell E, Wong WB, Hull W, Whitsett JA, Akino T, Kuroki Y, et al: Serial changes in surfactant-associated proteins in lung and serum before and after onset of ARDS. Am J Respir Crit Care Med 160: 1843-1850, 1999

21. Galani V, Tatsaki E, Bai M, Kitsoulis P, Lekka M, Nakos G and Kanavaros P: The role of apoptosis in the pathophysiology of Acute Respiratory Distress Syndrome (ARDS): An up-to-date cell-specific review. Pathol Res Pract 206: 145-150, 2010.
22. Zhang Y, Zhang B, Xu DQ, Li WP, Xu M, Li JH, Xie XY, Fan QX, Liu W, Mu DG, et al: Tanshinone IIA attenuates seawater aspiration-induced lung injury by inhibiting macrophage migration inhibitory factor. Biol Pharm Bull 34: 1052-1057, 2011.

23. Lee YJ, Choi DY, Yun YP, Han SB, Oh KW and Hong JT: Epigallocatechin-3-gallate prevents systemic inflammation-induced memory deficiency and amyloidogenesis via its anti-neuroinflammatory properties. J Nutr Biochem 24: 298-310, 2013

24. Lee IT, Lin CC, Lee CY, Hsieh PW and Yang CM: Protective effects of (-)-epigallocatechin-3-gallate against TNF- $\alpha$-induced lung inflammation via ROS-dependent ICAM-1 inhibition. J Nutr Biochem 24: 124-136, 2013.

25. Butt MS, Ahmad RS, Sultan MT, Nasir QM and Naz A: Green tea and anticancer perspectives: Updates from last decade. Crit Rev Food Sci Nutr 55: 792-805, 2015.

26. Wang P, Wang B, Chung S, Wu Y, Henning SM and Vadgama JV: Increased chemopreventive effect by combining arctigenin, green tea polyphenol and curcumin in prostate and breast cancer cells. RSC Advances 4: 35242-35250, 2014.

27. Singh M, Bhui K, Singh R and Shukla Y: Tea polyphenols enhance cisplatin chemosensitivity in cervical cancer cells via induction of apoptosis. Life Sci 93: 7-16, 2013.

28. An Z, Qi Y, Huang D, Gu X, Tian Y, Li P, Li H and Zhang Y: EGCG inhibits Cd(2+)-induced apoptosis through scavenging ROS rather than chelating $\mathrm{Cd}(2+)$ in HL-7702 cells. Toxicol Mech Methods 24: 259-267, 2014.

29. Shin ES, Huang Q, Gurel Z, Palenski TL, Zaitoun I, Sorenson CM and Sheibani N: STAT1-mediated Bim expression promotes the apoptosis of retinal pericytes under high glucose conditions. Cell Death Dis 5: e986, 2014. 